\title{
Capacity of Grid-Oriented Wireless Mesh Networks
}

\author{
Nadeem Akhtar and Klaus Moessner \\ Centre for Communication Systems Research \\ University of Surrey \\ Guildford, GU2 7XH, UK \\ Email: n.akhtar@surrey.ac.uk, k.moessner@surrey.ac.uk
}

\begin{abstract}
Wireless mesh networking technology has gained prominence in recent years with a diverse range of applications from small community-based networks to metropolitan area networks. Such mesh networks typically comprise a wireless backbone connected to the wired infrastructure with wireless client residing at the edge. The wireless routers and access points of a wireless mesh network (WMN) are connected by shared links that often use the same channel. The capacity of a WMN depends on several factors such as network size and topology, traffic profile, number of radio interfaces per node, number of radio channels, channel assignment scheme, routing algorithm etc. In this paper, we describe an analytical framework for capacity estimation using the notion of collision domains. Furthermore, we analyse the capacity of WMNs under various scenarios to study the impact of different network parameters.
\end{abstract}

\section{INTRODUCTION}

Wireless mesh networks have generated a lot of interest in recent years, both in the industry as well as the research community. These networks can potentially be used in a wide range of applications [1]. WMNs are deployed by Internet Service Providers for last-mile connectivity. In this case, some of the WMN nodes are directly connected to the fixed infrastructure via wired links thereby enabling the remaining nodes to get access via them using multi-hop wireless paths. In Metropolitan Area Network scenarios, the wired backhaul is replaced with city-wide WMNs using comprising wireless routers. For the case of enterprise networks, access points are connected together in mesh topology with a wireless backbone. In all these scenarios, replacement of wired infrastructure with WMN drives down the overall deployment cost and creates a more flexible network topology.

The most commonly used mesh architecture is where a wireless backbone is connected to the fixed infrastructure via one or more wired links. This is referred to as infrastructure or backbone WMN. Such a network comprises a set of nodes connected by wireless links, with one or more of the nodes directly connected to the global Internet. The latter are referred to as gateways. Nodes in a backbone WMN can be client devices, wireless access points and wireless routers. Wireless routers and access points are typically fixed and hence, WMNs made up entirely of such devices will have a stable topology whereas WMNs made up of client devices only will be dynamic in general. In the following, unless mentioned otherwise, the term WMN refers to backbone mesh networks comprising wireless access points and routers with one or more client nodes at the edge.
The design and deployment of a WMN for any application scenario is challenging because there are several interlinked factors that can impact the performance of the network. Some of them are network-related such as network topology, traffic profile etc. Other factors are radio related, for example, frequency band, channel bandwidth, number of available channels, number of radios per node etc. Several different metrics are used to quantify WMN performance including capacity, throughput, goodput, delay etc. Among these, capacity is undoubtedly one of the most important indicators. In this paper, we address the issue of capacity estimation of multiradio, multi-channel WMNs.

Capacity analysis of multi-hop wireless networks has proven to be an interesting and challenging research topic which is reflected in the wide body of related literature [2] [3] [4] [5] [6]. In [2], lower and upper bounds of network capacity were determined. This work also provided the important result that there is a significant decrease in throughput capacity per node as node density increases. However, the analysis does not capture routing-related effects and assumes that all paths follow straight lines. Thus, it can only be applied to WMNs in which nodes are organised in a chain topology. A theoretical framework for determining the nominal capacity of WMNs was described in [7]. The concept of collision domains is used to determine the bottleneck link in the network and upper bound on capacity calculated. The analytical model was validated using simulations. However, only single channel WMNs were considered.

The capacity of WMNs depends on the available radio capacity. Given the fact that, in most cases, nodes in backbone WMN are dedicated wireless routers and access points, it is reasonable to assume that these nodes have multiple radios thereby enabling use of multiple channels. An important aspect of multi-radio multi-channel mesh networks is the assignment of radio channels to interfaces. [8] [9] propose an integrated channel assignment and routing algorithm for multi-radio multi-channel WMNs. An iterative algorithm based on greedy channel assignment is used. However, the topology used in this study had ingress-egress node pairs distributed randomly. This maybe true when the WMN is operating in an ad-hoc networking scenario but in backbone WMNs, the ingressegress pairs are not randomly distributed. A new routing metric for multi-radio WMNs was defined in [10] to capture loss rate and link bandwidth. This metric is then used to select the best paths for routing packets. 
Although, the research cited above studied the capacity of WMNs, the scenarios used tend to be rather specific. There is a need to determine WMN capacity for a wider range of scenarios so as to provide insights into the impact of different design factors such as network topology, network size, routing methods, channel assignment schemes etc on the capacity of WMNs. In this paper, the relationships between these design parameters and the capacity of multi-radio, multichannel WMNs are explored.

The rest of this paper is organised as follows. In the next section, we briefly explain the notion of collision domains. It is followed by the description of an analytical framework to estimate WMN capacity. We estimate the capacity of WMNs for different scenarios in Section IV. Finally, Section $\mathrm{V}$ contains the main conclusions from this work.

\section{Collision Domains}

Collision domains arise due to the shared nature of wireless links that connect WMN nodes. Use of a common channel implies that when a node is transmitting, all other nodes in its immediate vicinity must not transmit at the same time, assuming that there are no other means of providing isolation between simultaneous transmissions. Consider a wireless network with $\mathrm{N}$ nodes and the associated graph $\mathrm{G}(\mathrm{V}, \mathrm{E})$, where $\mathrm{V}$ is the set of nodes and $\mathrm{E}$ is the set of wireless links connecting the nodes. A virtual wireless link exists between a pair of nodes $i$ and $j$ if the distance between them is less than or equal to the transmission range of the nodes.

The collision domain $C_{i j}$ corresponding to link $l_{i j}$ is the set of links consisting of $l_{i j}$ and all other links that must be inactive for collision-free transmissions between nodes $i$ and $j$. The size of a collision domain is directly related to the interference range of wireless nodes. $C_{i j}$ will contain link $l_{i j}$ and all other links that terminate on nodes within the interference range of either node $i$ or $j$.

Figure 1 shows 7 nodes organised in chain topology, connected via wireless links. Adjacent nodes are assumed to be just within the transmission range of each other. Furthermore, the interference range is assumed to be twice the transmission range. Consider link $l_{45}$ which connects nodes 4 and 5. Other links that have at least one of their end nodes within the interference range of nodes 4 or 5 must be inactive whilst link $l_{45}$ is active, as indicated by the dashed arrows in Figure 1. All the links located inside the dashed region are part of the collision domain of link $l_{45}$. Similarly, the collision domains of other links can be determined by identifying the corresponding transmission constraints. For example, the collision domain of $l_{12}$ consists of $l_{12}, l_{23}$ and $l_{34}$.

The maximum capacity of each collision domain is limited by nominal MAC throughput and hence, there exists a collision domain which creates an upper bound on the network capacity. A bottleneck collision domain is defined as the one that needs to forward the maximum amount of traffic [7]. It is possible that a network may have more than one bottleneck domain. In the following, we present an analytical framework to determine

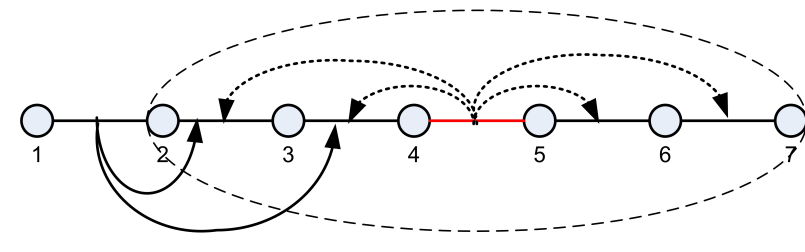

Fig. 1. Collision domain

the bottleneck collision domain(s) of a WMN which is then used to estimate the nominal capacity of the network.

\section{Analytical Model}

The model presented in [7] uses nominal MAC throughput and the concept of collision domains for estimating capacity of wireless mesh networks. The former is defined as the throughput achieved at the MAC layer in a one-hop IEEE 802.11 network operating in infrastructure mode. It depends on a number of factors such as MAC layer characteristics, channel conditions, network topology and packet size distribution etc and can be determined if the relevant parameters are known [11].

Consider a network of $\mathrm{N}$ wireless nodes represented by the graph $G(V, E)$ where $V$ is the set of nodes and $E$ is the set of links between these nodes. Each node is assumed to have $p$ radio interfaces and $q$ available channels. An arbitrary channel assignment algorithm allocates channels to radio interfaces of each network node. One of the network nodes is designated as gateway. Furthermore, $K$ out of the remaining $N-1$ nodes generate application traffic with the gateway node as destination. These are referred to as aggregator nodes as they aggregate traffic from mesh clients and forward it towards the gateway. A routing protocol establishes end-to-end paths between each traffic generator and the gateway node.

The first step is to calculate the expected load on each link in the network. Let $b_{i}$ be the rate of traffic generated by node $i$. The expected load on an arbitrary link $l$ in the WMN is given by

$$
\phi_{l}=\sum_{i=1}^{K} b_{i} * \lambda_{i, l}
$$

where $\lambda_{i, l}$ is a binary variable whose value is 1 if the traffic originating from node $i$ traverses link $l$, otherwise it is zero.

The next step is to determine all collision domains of the network. In a multi-channel mesh network, the collision domain $C_{l}$ of a link $l$ consists of that link and all other links that fall within its interference range and share the same channel. Given the set of collision domains $\mathrm{C}, C_{l}$ is a bottleneck collision domain if

$$
\sum_{i \in C_{l}} \phi_{i}=\max _{C_{j} \in C} \sum_{k \in C_{j}} \phi_{k}
$$

We denote the collision domain as $C_{B}$. Note that, in general, there may be more than one bottleneck collision domain in a network. Furthermore, in a homogeneous network, where the available bandwidth of all domains is same, the bottleneck 
domain is the one with the highest expected load. The expected load on the bottleneck domain cannot exceed the nominal MAC capacity, $B$. Therefore,

$$
\sum_{j \in C_{B}} \phi_{j} \leq B
$$

The right-hand side of this inequality can be expressed as the weighted sum of the ingress flow rates $b_{i}$. The exact relationship depends on the routing and channel assignment algorithms. In the analysis that follows, it is assumed that the steady state rate of the flows emanating from the aggregator nodes is same. Therefore, Equation 1 can be simplified as below

$$
\phi_{l}=b * \sum_{i=1}^{K} \lambda_{i, l}
$$

where $b$ is the flow rate of an aggregator node.

Using this in Equation 3 yields

$$
b * \sum_{j \in C_{B}} \alpha_{j} \leq B
$$

where $\alpha_{j}$ is given by

$$
\alpha_{j}=\sum_{i=1}^{K} \lambda_{i, l}
$$

Therefore,

$$
b \leq \frac{B}{\sum_{j \in C_{B}} \alpha_{j}}
$$

Hence,

$$
b_{\max }=\frac{B}{\sum_{j \in C_{B}} \alpha_{j}}
$$

It must be noted that this holds true only under the assumption that absolute fairness is enforced in the network to ensure that the available bandwidth is equally shared between nodes. In [7], this method was validated by simulation and the observed capacity closely matched the theoretical results.

Note that the assumption of a single gateway is used only as a simplification. The same method can be used for multigateway WMNs as well. The presence of multiple gateways will obviously affect the expected link loads but the procedure for identifying the collision domains remains the same.

\section{CAPACITY OF GRID-ORIENTED WMNS}

We used the analytical framework described above to estimate the nominal capacity of WMNs organised in the form of a 2-dimensional grid for a wide range of scenarios characterised by different grid sizes, number of aggregator nodes, routing algorithms and channel assignment schemes. In the following, first we describe the scenarios used for the analysis and then present the results.

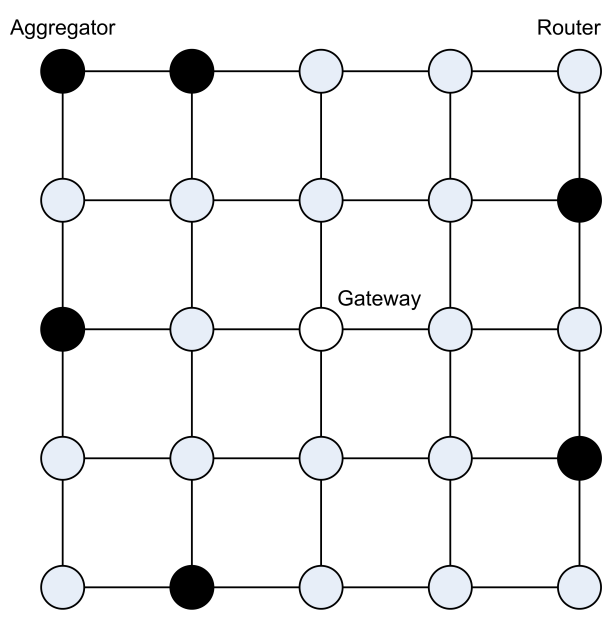

Fig. 2. Grid WMN Topology

\section{A. Scenario Synthesis}

Note that the topology of a WMN ultimately depends on the deployment scenario but some features are common. There are one or more gateway nodes with connectivity to the global Internet via wired links while the remaining nodes are wireless routers or access points (or hybrid nodes that can work as both). Access points act as aggregators of traffic originating from client nodes attached to them.

Grid-oriented WMNs have been widely used in literature for performance analysis. Although it is not realistic to assume that grid-based WMNs can be used in all scenarios, it is reasonable to expect them to be used, at least, in urban environments. Note that grids can also be used to generate more random topologies by removing some nodes. Bearing these considerations in mind, the analysis here focusses on WMNs organised in grid formation. Furthermore, it is assumed that there is only one gateway node. The topology considered for capacity analysis is shown in Figure 2 where the gateway node is located at the center of the grid and other nodes are located at remaining grid points. The aggregators are distributed along the grid boundary and wireless routers can be located at the edges as well as interior grid points.

The topology described above has a number of aggregator nodes generating traffic with the gateway node as the destination. As seen from Figure 2, multiple paths are available between a given aggregator node and the gateway. These paths can be utilised in many ways. One option is multipath routing where all available paths are used simultaneously by distributing the traffic over them. Another multipath routing strategy is to shortlist a few of the available routes based on some suitable criteria and distribute the traffic over this subset of paths. A special case is the single path approach in which only one of the available routes is selected and all the traffic is forwarded along it. In our analysis, we consider only the single path routing algorithm which selects the shortest available path from a given aggregator node to the gateway.

Channel assignment is another important aspect of the 
scenario. Single-radio WMNs can use only one channel even if several are available to ensure that the network does not become partitioned. However, in multi-radio WMNs, it is possible to utilise multiple channels simultaneously. In such scenarios, channels must be assigned to radio interfaces in such a way that there is at least one path between every pair of nodes to avoid partitioning. Furthermore, the assignment must also take into account performance objectives such as interference reduction, fair distribution of load and throughput maximisation.

Three different channel assignment schemes are considered. The first is relatively simple where all interfaces are assigned the same channel. This is essentially a baseline case against which other cases are compared. In the second scheme, the partitioning algorithm proposed in [8] is used for uniform channel assignment across the network. Assuming that each node has $k$ interfaces, the algorithm starts by selecting one node and dividing its neighbours into $k$ groups. Each interface of the node is assigned a group. In the next iteration, each neighbour node is similarly partitioned without violating the grouping established earlier. The process is repeated until all the nodes have executed the procedure. Finally, each group is assigned the least-used channel in the neighbourhood. Figure 3 shows the result of this channel assignment scheme for a $5 \times 5$ grid WMN with $k=2$ and 6 available channels. Numbers along the lines indicate the channel assigned to the corresponding link. The third scheme assigns channels to interfaces based on the condition that available bandwidth on the interfaces is at least equal to the expected load on the associated links [8]. The scheme uses a greedy load-aware algorithm where channels are assigned to links one-by-one in the decreasing order of expected load. For a given network topology and knowing the amount of traffic generated by each aggregator node, the expected load on each link can be calculated from the routing pattern using Equation 1. When a link is traversed, the channel assigned to it is such that it results in the least amount of interference from other links that are in its collision domain. Since links with higher loads are visited first, it is expected that they will be allocated a channel that suffers from less interference and therefore, get a greater share of the available bandwidth.

The different aspects described above have been combined to generate several scenarios. The following assumptions are applicable to all scenarios:

1) All nodes on the grid are stationary;

2) Traffic in the network is generated by aggregator nodes only and all other nodes, including wireless routers and the gateway, do not generate any traffic;

3) Traffic generated by aggregator nodes is destined for the gateway;

4) Interference range is assumed to be 2 hops.

The last condition implies that nodes that are within 2 hops distance of a particular node will cause interference to it if one or more of their interfaces use the same channel. In addition, the assumptions mentioned in [7] also apply here. In particular,

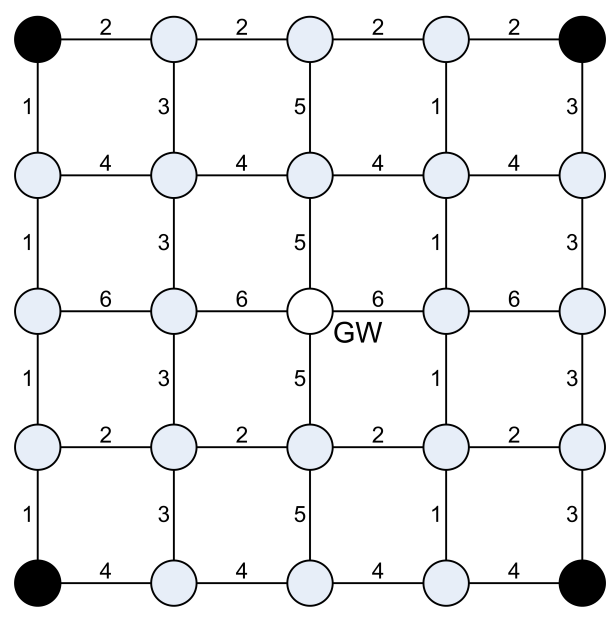

Fig. 3. Channel assignment based on partitioning

absolute fairness is assumed. Furthermore, the MAC layer supports Request-To-Send/Clear-To-Send (RTS/CTS) handshake mechanism resulting in symmetric link constraints for collision domain determination.

\section{B. Results}

The capacity of WMNs for the scenarios presented above was estimated by implementing the analytical model in MATLAB. The WMN nodes were assumed to have two radio interfaces. Furthermore, two values were used for the number of available channels -3 and 12 . The former corresponds to the number of non-overlapping channels in IEEE $802.11 \mathrm{~b}$ while the latter is the number of channels available in IEEE802.11a. A $5 \times 5$ grid is considered here, unless otherwise stated. All the aggregator nodes in each scenario generate the same amount of traffic. Without loss of generality, the nominal MAC capacity is assumed to be 1 and the capacity available to each WMN node is calculated as percentage of the nominal MAC capacity. The capacity is computed for varying number of aggregator nodes, which are randomly placed on the grid points located along the boundary. In most cases, there are many ways to distribute a given number of aggregator nodes on a fixed set of grid points. Therefore, the analysis considers a large number of possible node placements and averages over them.

The results presented here show capacity as percentage of nominal MAC throughput. Note that each data point is the average over 1000 runs (for the same number of aggregator nodes). During each run, a given number of aggregators is randomly placed on the set of available grid points. We use the single channel scenario as the baseline case.

The following notation is used in the graphs:

- C: Channel assignment scheme (scheme 1 is uniform assignment, scheme 2 is partitioning-based assignment and scheme 3 is load-aware assignment)

- c: Number of available channels

- K: Number of aggregator nodes

Figure 4 shows the nominal capacity of the network when 3 and 12 channels are available, respectively. The results clearly 

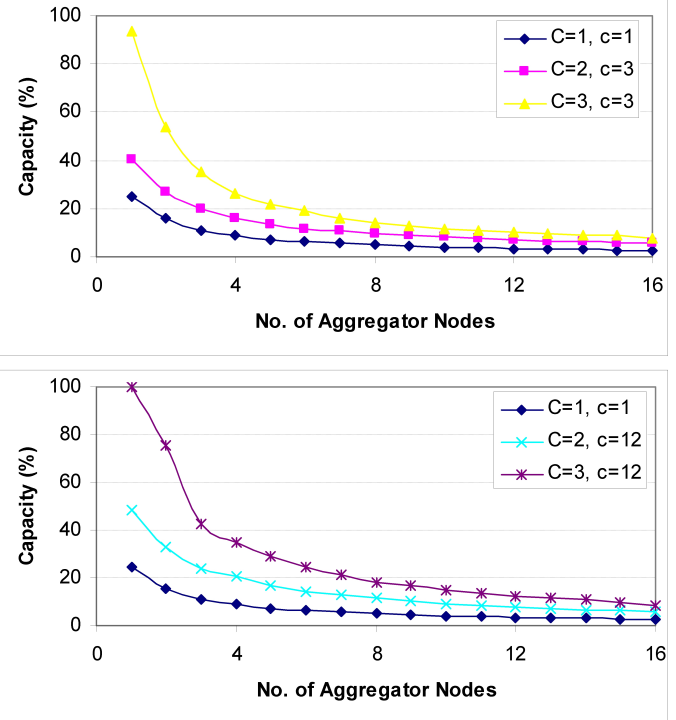

Fig. 4. Capacity vs Traffic Load for Fixed Grid Size

indicate that the capacity available to each aggregator node increases when multiple channels are used. For the baseline case where only one aggregator is present, the maximum throughput is only around $25 \%$ of the nominal MAC capacity when a single channel is available. In contrast, when multiple channels are used in conjunction with the partitioning-based channels assignment scheme, the capacity is much higher, close to $40 \%$ when 3 channels are used and 50\% when 12 channels are available. Furthermore, the load-aware assignment scheme results in greater gains with the capacity exceeding $90 \%$ and reaching $100 \%$ for 3 and 12 channels scenarios respectively. As the number of aggregator nodes increases, the differences between the 3 assignment schemes gradually diminish but the general trend stays the same. In other words, the channel assignment scheme appears to be an important factor in determining the capacity. On the whole, load-aware channel assignment outperforms the partitioningbased method. The results also show that capacity falls sharply as the number of aggregator nodes increases.

The next set of results illustrate the effect of grid size on capacity. In this case, we consider square grids of different sizes and compute the capacity for different number of aggregator nodes (K). Two different cases are considered. First, we fix K and vary the grid size and repeat this for different values of $\mathrm{K}$. Next, we fix the aggregator node density and vary the grid size. For an $N \times N$ grid, the maximum number of aggregator nodes that can be deployed on the grid edges is $4(N-1)$. The aggregator density is defined as

$$
\rho=\frac{K}{4(N-1)} * 100
$$

Figures 5 and 6 show the capacity variation with grid size for these two cases when 3 and 12 channels are used, respectively. We observe that the grid size does not seem to
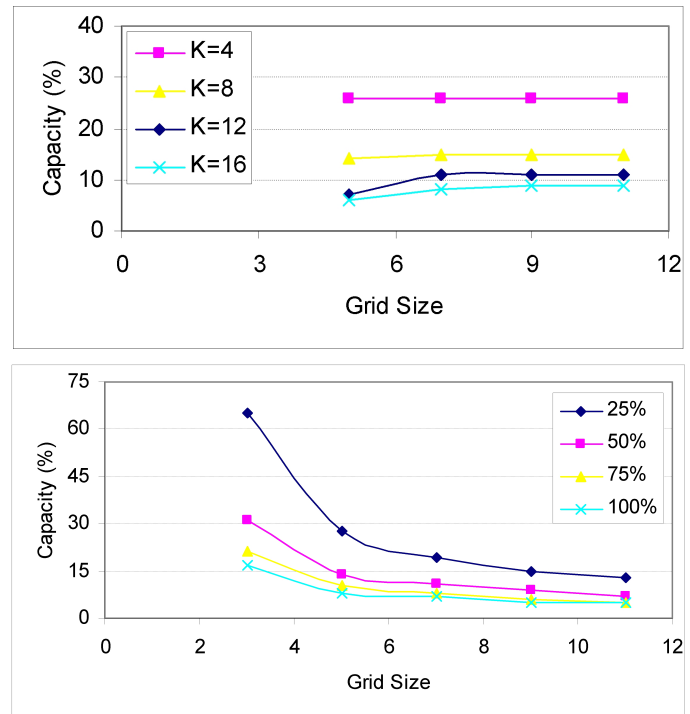

Fig. 5. Impact of Grid Size and Aggregator Density $(c=3)$
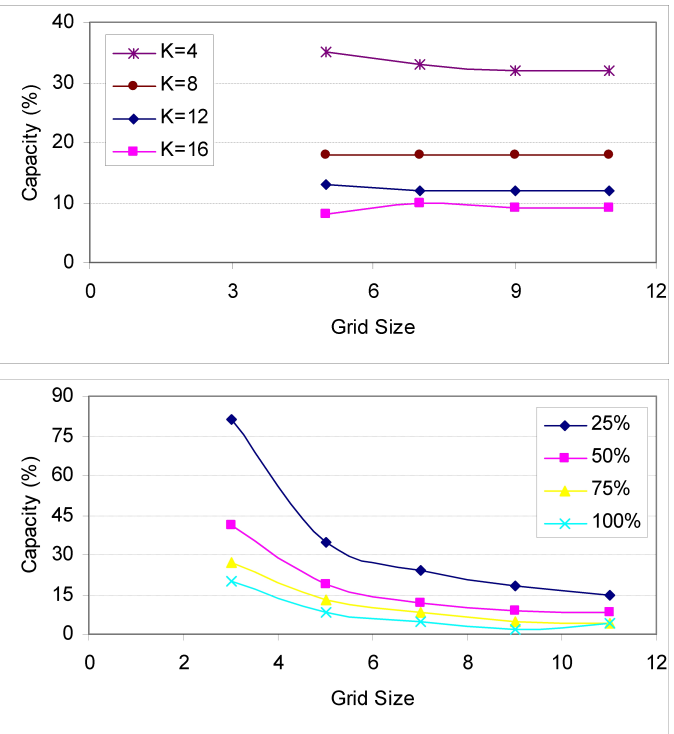

Fig. 6. Impact of Grid Size and Aggregator Density $(c=12)$

have much effect on capacity when the number of aggregator nodes is fixed. In contrast, when we fix the aggregator density, capacity falls rapidly with grid size before reaching a saturation point.

\section{Discussion}

The results presented above provide insights on the impact of different WMN design parameters on the available capacity at each aggregator node. In the following, we discuss some of these implications.

There is an inverse relationship between the number of aggregator nodes and capacity. The main reason for this is the increase in expected load with number of aggregators which 
means that each collision domain has to carry greater load on the average, thereby decreasing the per-node capacity. Even for a given number of aggregator nodes, the capacity depends on the placement of these nodes with respect to each other. In particular, if aggregators are placed close together, then a small subset of links will have to carry all the traffic load and hence, capacity will be low. On the other hand, when separation between nodes is large, load is distributed more evenly in different parts of the grid, thereby resulting in higher capacity. Note that it is possible to provide good separation when the number of aggregators is small but the same cannot be said for scenarios with large number of aggregators.

The location of gateway node with respect to aggregator nodes also influences the capacity. As the size of grid increases, the average distance between aggregators and the gateway also increases. As a result, routes between aggregators and gateway traverse through more links and hence, overall interference levels go up. However, increasing the grid size also implies that a given number of aggregator nodes can have greater separation between them which leads to reduced interference. These two effects cancel each other out and hence, the results show very small decrease in capacity with increasing grid size when the number of aggregator nodes is independent of grid size. In contrast, when the grid size is varied for while keeping the aggregator density fixed, we see that capacity falls sharply. The reason for this behaviour lies in the effect that the number of aggregator nodes increases when grid size is increased in order to maintain the same density. Therefore, the increase in separation between aggregator nodes is much smaller and hence the dominant effect is that of long routes sharing many links.

The effect of increasing the number of available channels is significant when the number of aggregator nodes is not too high with respect to the available locations on the grid boundary. On the average, when the number of aggregator nodes is low, increasing the number of channels from 1 to 3 results in capacity gain of $10-20 \%$, depending on the channel assignment schemed used. For the IEEE802.11 a case, capacity improvements is in the $10-30 \%$ range in most cases when the number of channels is increased from 1 to 12 . However, for high load scenarios, there is very little improvement for multichannel scenarios. Even though a relatively large number of channels are available, since the number of wireless interfaces is just 2, the ability of the network to use all the available channels is limited. The load-aware channel assignment algorithm performs better compared to the partitioning-based method, especially when the number of aggregators is low to moderate. However, the gains are limited by the number of radio interfaces available. The results, therefore, indicate that using multiple radios in conjunction with load-aware channel assignment improves capacity of multi-channel WMNs.

\section{CONCLUSiOns}

In this paper, we presented an analytical framework for determining the nominal capacity of multi-radio multi-channel wireless mesh networks. The objective was to study the impact of various network design parameters on WMN capacity. The notion of collision domain is used to calculate an upper bound on the capacity available to ingress nodes that generate traffic towards the node that acts as the WMN gateway to the outside world.

The results presented here indicate that the effects of WMN design parameters are interlinked and a judicious selection is essential to maximise capacity. Use of multiple channels improves capacity as neighbouring links can be used simultaneously. However, the reuse factor depends on the number of radio interfaces. As a result, when the number of channels is increased from 3 to 12 , the resulting capacity gain is not that high. Furthermore, increasing the number of aggregator nodes also limits the capacity improvement significantly. Grid size appears to have little effect on capacity when the number of aggregator nodes is fixed but capacity falls when the grid size is increased while keeping the aggregator node density fixed.

\section{REFERENCES}

[1] I. F. Akyilidz, X. Wang, and W. Wang, "Wireless Mesh Networks: A Survey," in Elsevier Computer Networks Journal, vol. 47, March 2005, pp. $445-487$.

[2] P. Gupta and P. R. Kumar, "The Capacity of Wireless Networks," in IEEE Trans. Information Theory, March 2000, vol. 46 no. 2, pp. 388404.

[3] J. Li, C. Blake, D. S. J. D. Couto, H. I. Lee, and R. Morris, "Capacity of Ad Hoc Wireless Networks," in Proceeding of 7th ACM International Conference on Mobile Computing and Networking, July 2001, pp. 6169.

[4] M. Grossglauser and D. Tse, "Mobility Increases the Capacity of Ad Hoc Wireless Networks," in IEEE/ACM Trans. Networking, August 2002, vol. 10 no. 4, pp. 477-486.

[5] M. Gastpar and M. Vetterli, "On the Capacity of Wireless Networks: The Relay Case," in Proceeding of IEEE INFOCOM 2002, vol. 3, June 2002, pp. 1577-1586.

[6] F. Cali, M. Conti, and E. Gregori, "IEEE 802.11 Wireless LAN: Capacity Analysis and Protocol Enhancement," in Proceedings of IEEE INFOCOM 1998, vol. 1, March 1998, pp. 142-149.

[7] J. Jangeun and M. L. Sichitiu, "The Nominal Capacity of Wireless Mesh Networks," in IEEE Wireless Communications Magazine, October 2003, vol. 10 no. 5 , pp. 8-14.

[8] A. Raniwala, K. Gopalan, and T. cker Chiueh, "Centralized Channel Assignment and Routing Algorithms for Multi-channel Wireless Mesh Networks," in ACM SIGMOBILE Mobile Computing and Communication Review, April 2004, vol. 8 no. 2, pp. 50-65.

[9] A. Raniwala and T. cker Chiueh, "Architecture and Algorithms for an IEEE 802.11-based Multi-channel Wireless Mesh Network," in Proceedings of INFOCOM 2005, March 2005, vol. 3, pp. 2223-2234.

[10] R. Draves, J. Padhye, and B. Zill, "Routing in Multi-radio, Multi-hop Wireless Mesh Networks," in Proceedings of ACM MobiCom 2004, September 2004, pp. 114-128.

[11] J. Jun, P. Peddabachagari, and M. L. Sichitiu, "Theoretical Maximum Throughput of IEEE 802.11 and Its Applications," in Proceedings of 2nd IEEE International Symposium on Network Computing and Applications, April 2003, pp. 259-256. 